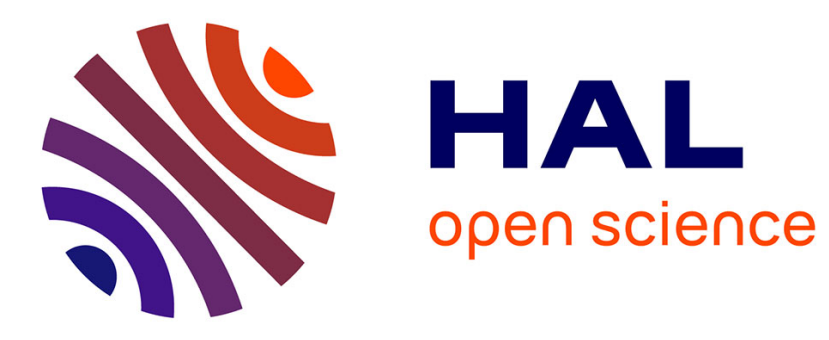

\title{
On a non-archimedean broyden method
}

\author{
Xavier Dahan, Tristan Vaccon
}

\section{To cite this version:}

Xavier Dahan, Tristan Vaccon. On a non-archimedean broyden method. ISSAC '20: International Symposium on Symbolic and Algebraic Computation, Jul 2020, Kalamata Greece, France. pp.114-121, 10.1145/3373207.3404045 . hal-02928697

\section{HAL Id: hal-02928697 https://hal.science/hal-02928697}

Submitted on 2 Sep 2020

HAL is a multi-disciplinary open access archive for the deposit and dissemination of scientific research documents, whether they are published or not. The documents may come from teaching and research institutions in France or abroad, or from public or private research centers.
L'archive ouverte pluridisciplinaire HAL, est destinée au dépôt et à la diffusion de documents scientifiques de niveau recherche, publiés ou non, émanant des établissements d'enseignement et de recherche français ou étrangers, des laboratoires publics ou privés. 


\section{On A Non-Archimedean Broyden Method}

\author{
Xavier Dahan \\ Tohoku University, IEHE \\ Sendai, Japan 980-8576 \\ xdahan@gmail.com
}

\author{
Tristan Vaccon \\ Université de Limoges; CNRS, XLIM UMR 7252 \\ Limoges, France 87060 \\ tristan.vaccon@unilim.fr
}

\begin{abstract}
Newton's method is an ubiquitous tool to solve equations, both in the archimedean and non-archimedean settings - for which it does not really differ. Broyden was the instigator of what is called "quasiNewton methods". These methods use an iteration step where one does not need to compute a complete Jacobian matrix nor its inverse. We provide an adaptation of Broyden's method in a general nonarchimedean setting, compatible with the lack of inner product, and study its $\mathrm{Q}$ and $\mathrm{R}$ convergence. We prove that our adapted method converges at least Q-linearly and R-superlinearly with R-order $2 \frac{1}{2 m}$ in dimension $m$. Numerical data are provided.
\end{abstract}

\section{KEYWORDS}

System of equations, Broyden's method, Quasi-Newton, p-adic approximation, Power series, Symbolic-numeric, p-adic algorithm

\section{ACM Reference format:}

Xavier Dahan and Tristan Vaccon. 2020. On A Non-Archimedean Broyden Method. In Proceedings of The 45th International Symposium on Symbolic and Algebraic Computation, Kalamata, Greece, fuly 2020 (ISSAC'20), 9 pages. DOI:

\section{INTRODUCTION}

In the numerical world. Quasi-Newton methods refer to a class of variants of Newton's method for solving square nonlinear systems, with the twist that the inverse of the Jacobian matrix is "approximated" by another matrix. When compared to Newton's method, they benefit from a cheaper update at each iteration (See e.g. [10, p.49-50,53]), but suffer from a smaller rate of convergence. They were mainly introduced by Broyden in [6], which has sparked numerous improvements, generalizations, and variants (see the surveys $[10,19])$. It is now a fundamental numerical tool (that finds its way in entry level numerical analysis textbooks [8, § 10.3]). To some extent, this success stems from: the specificities of machine precision arithmetic as commonly used in the numerical community, the fact that Newton's method is usually not quadratically convergent from step one, and that the arithmetic cost of an iteration is independent of the quality of the approximation reached. In another direction, variants of Broyden's method have known dramatic success for unconstrained optimization - the target system is the gradient of the objective function, the zeros are then critical points- where it takes advantage of the special structure of the

Permission to make digital or hard copies of part or all of this work for personal or classroom use is granted without fee provided that copies are not made or distributed for profit or commercial advantage and that copies bear this notice and the full citation on the first page. Copyrights for third-party components of this work must be honored. For all other uses, contact the owner/author(s).

ISSAC'20, Kalamata, Greece

(C) 2020 Copyright held by the owner/author(s). ...\$15.00 DOI:
Hessian (see Sec. 7 of [10]). Another appealing feature of Broyden's method is the possibility to design derivative-free methods generalizing to the multivariate case the classical secant method (which can be thought of as Broyden's in dimension one). This feature is a main motivation for this work.

Non-archimedean. It is a natural wish to transpose such a fundamental numerical method to the non-archimedean framework, offering new tools to perform exact computations, typically for systems with $p$-adic or power series coefficients. For this adaptation, several non-trivial difficulties have to be overcome: e.g. no inner products, a more difficult proof of convergence, or a management of arithmetic at finite precision far more subtle. This article presents satisfactory solutions for all these difficulties, which we believe can be expanded to a broader variety of quasi-Newton methods.

Bach proved in [1] that in dimension one, the secant method can be on an equal footing with Newton's method in terms of complexity. We investigate how this comparison is less engaging in superior dimension (see Section 6). To our opinion, this is due to the remarkable behavior of Newton's method in the non-archimedean setting. No inversion of the Jacobian is required at each iteration (simply a matrix multiplication, this is now classical see $[5,16,17])$. The evaluation of the Jacobian is also efficient for polynomial functions (in dimension $m$, it involves only $O(m)$ evaluations, instead of $m^{2}$ over $\mathbb{R}$, see [2]). It displays also true quadratic behavior from step one which, when combined with the natural use of finite precision arithmetic (against machine precision over $\mathbb{R}$ ), offers a ratio cost/precision gained that is hard to match.

And indeed, our results show that for large dimension $m$ and polynomials as input, there is little hope for Broyden to outperform Newton, although it depends on the order of superlinear convergence of Broyden's method. In this respect more investigation is necessary, but for now the interest lies more in the theoretical advances and in the situations mentioned in "Motivations" thereafter.

Relaxed arithmetic. Since the cost of one iteration of Broyden's method involves $m^{2}$ instead of $m^{\omega}$ for Newton, we should mention the relaxed framework (a.k.a online [11]) which show essentially the same decrease of complexity, while maintaining quadratic convergence. It has been implemented efficiently for power series [23], and for $p$-adic numbers [3]. In case of a smaller $m$ and a larger precision of approximation required, FFT trading [24] has to be mentioned. These techniques are however unlikely to be suited to the Broyden iteration, since it is a priori not described by a fixed-point equation, a necessity for the relaxed machinery.

Motivations. As explains Remark 6.4, it seems unlikely in the non-archimedean world that with polynomials or rational fractions, a quasi-Newton method meets the standard of Newton's method. The practical motivations concern:

1/ Derivative-free method: instead of starting with the Jacobian at precision one, use a divided-difference matrix. A typical application 
is when the function is given by a "black-box" and there is no direct access to the Jacobian.

2/ When computing the Jacobian does not allow shortcuts like in the case of rational fractions [2], evaluating it may require up to $L m^{2}$ operations, where $L$ is the complexity of evaluation of the input function. Regarding the complexity of Remark 6.4, Broyden's method then becomes beneficial when $L \gtrsim m^{2}-m^{\omega-1}$.

3/ While Newton's method over general Banach spaces of infinite dimension can be made effective when the differential is effectively representable (integral equations $[15, \S 5][14]$ are a typical example), it is in general difficult or impossible to compute it. On the other hand, Broyden's method or its variants have the ability to work with approximations of the differential, including of finite rank, by considering a projection (as shown in [14, 15] and the references therein; the dimension of the projection is increased at each iteration). In the non-archimedean context, ODEs with parameters, for example initial conditions, constitute a natural application.

Organization of the paper. Definitions and notations are introduced in Section 2. Section 3 explains how Broyden's method can be adapted to an ultrametric setting. In Section 4, we study the $Q$ and R-order of convergence of Broyden's method (see Definition 2.1), presenting our main results. It is followed by Section 5, where are introduced developments and conjectures on Q-superlinearity. Finally, in Section 6, we explain how our Broyden's method can be implemented with dynamical handling of the precision, and we conclude with some numerical data in Section 7.

\section{BROYDEN'S METHOD AND NOTATIONS}

\subsection{General notations}

Throughout the paper, $K$ refers to a complete, discrete valuation field, val $: K \rightarrow \mathbb{Z} \cup\{+\infty\}$ to its valuation, $O_{K}$ its ring of integers and $\pi$ a uniformizer. ${ }^{1}$ For $k \in \mathbb{N}$, we write $O\left(\pi^{k}\right)$ for $\pi^{k} O_{K}$.

Let $m \in \mathbb{Z}_{\geq 1}$. We are interested in computing an approximation of a non-singular zero $x^{\star}$ of $f: K^{m} \rightarrow K^{m}$ through an iterative sequence of approximations, $\left(x_{n}\right)_{n \in \mathbb{N}} \in\left(K^{m}\right)^{\mathbb{N}}$. Note that all our vectors are column-vectors. For any $x \in K^{m}$ where it is welldefined, we denote by $f^{\prime}(x) \in M_{m}(K)$ the Jacobian matrix of $f$ at $x$. We will use the following notations (borrowed from [13]):

$$
f_{n}=f\left(x_{n}\right), \quad y_{n}=f_{n+1}-f_{n}, \quad s_{n}=x_{n+1}-x_{n}
$$

We denote by $\left(e_{1}, \ldots, e_{m}\right)$ the canonical basis of $K^{m}$. In $K^{m}, O\left(\pi^{k}\right)$ means $O\left(\pi^{k}\right) e_{1}+\cdots+O\left(\pi^{k}\right) e_{m}$.

Newton's iteration produces a sequence $\left(x_{n}\right)_{n \in \mathbb{N}}$ given by:

$$
x_{n+1}=x_{n}-f^{\prime}\left(x_{n}\right)^{-1} \cdot f\left(x_{n}\right) \text {. }
$$

For quasi-Newton methods, the iteration is given by:

$$
x_{n+1}=x_{n}-B_{n}^{-1} \cdot f\left(x_{n}\right), \quad\left(\Rightarrow s_{n}=-B_{n}^{-1} \cdot f_{n}\right)
$$

with $B_{n}$ presumably not far from $f^{\prime}\left(x_{n}\right)$. More precisely, it is a generalization of the design of the secant method over $K$ where one approximates $f^{\prime}\left(x_{n}\right)$ by $\frac{f\left(x_{n}\right)-f\left(x_{n-1}\right)}{x_{n}-x_{n-1}}$. In quasi-Newton, it is thus required that:

$$
B_{n} \cdot\left(x_{n}-x_{n-1}\right)=f\left(x_{n}\right)-f\left(x_{n-1}\right) \quad\left(\Rightarrow B_{n} \cdot s_{n-1}=y_{n-1}\right)
$$

By this condition alone, $B_{n}$ is obviously underdetermined. To mitigate this issue, $B_{n}$ is taken as a one-dimensional modification of

${ }^{1}$ Discrete valuation is only needed in Section 6. For the rest complete and ultrametric is enough.
$B_{n-1}$ satisfying (2). Concretely, a sequence $\left(u_{n}\right)_{n \in \mathbb{N}} \in\left(K^{m}\right)^{\mathbb{N}}$ is introduced such that:

$$
\begin{aligned}
B_{n} & =B_{n-1}+\left(y_{n-1}-B_{n-1} s_{n-1}\right) \cdot u_{n-1}{ }^{t} . \\
1 & =u_{n-1}{ }^{t} \cdot s_{n-1} .
\end{aligned}
$$

In Broyden's method over $\mathbb{R}, u_{n-1}$ is defined by:

$$
u_{n-1}=\frac{s_{n-1}}{s_{n-1}{ }^{t} \cdot s_{n-1}} \text {. }
$$

The computation of the inverse of $B_{n}$ can then be done using the Sherman-Morrison formula (see [22]):

$$
B_{n}^{-1}=B_{n-1}^{-1}+\frac{\left(s_{n-1}-B_{n-1}^{-1} y_{n-1}\right) \cdot s_{n-1}{ }^{t} B_{n-1}^{-1}}{s_{n-1}{ }^{t} B_{n-1}^{-1} y_{n-1}} .
$$

This formula gives rise to the so-called "good Broyden's method". Using [22] provides the following alternative formulae:

$$
\begin{aligned}
& B_{n}=B_{n-1}+f_{n} \cdot u_{n-1}{ }^{t} . \\
& B_{n}^{-1}=B_{n-1}^{-1}-\frac{B_{n-1}^{-1} f_{n} \cdot u_{n-1}{ }^{t} B_{n-1}^{-1}}{u_{n-1} B_{n-1}^{-1} y_{n-1}} .
\end{aligned}
$$

\subsection{Convergence}

We recall some notions on convergence of sequences commonly used in the analysis of the behavior of Broyden's method.

Definition 2.1 ([20] Chapter 9). A sequence $\left(x_{k}\right)_{k \in \mathbb{N}} \in\left(K^{m}\right)^{\mathbb{N}}$ has $Q$-order of convergence $\mu \in \mathbb{R}_{>1}$ to a limit $x^{\star} \in K^{m}$, if:

$$
\exists r \in \mathbb{R}_{+}, \quad \forall k \text { large enough, } \quad \frac{\left\|x_{k+1}-x^{\star}\right\|}{\left\|x_{k}-x^{\star}\right\|^{\mu}} \leq r .
$$

If we can take $\mu=1$ and $r<1$ in the previous inequality, we say that $\left(x_{k}\right)_{k \in \mathbb{N}}$ has $Q$-linear convergence. For $\mu=2$, we say it has $Q$-quadratic convergence. The sequence is said to have $Q$-superlinear convergence if

$$
\lim _{k \rightarrow+\infty} \frac{\left\|x_{k+1}-x^{\star}\right\|}{\left\|x_{k}-x^{\star}\right\|}=0 .
$$

It is said to have $R$-order of convergence ${ }^{2} \mu \in \mathbb{R}_{\geq 1}$ if

$$
\lim \sup \left\|x_{k}-x^{\star}\right\|^{1 / \mu^{k}}<1 \text {. }
$$

Remark 2.2. For both $\mathrm{Q}$ and $\mathrm{R}$, we write has convergence $\mu$ to mean has convergence at least $\mu$.

Broyden's method satisfies the following convergence results:

THeORem 2.3. Over $\mathbb{R}^{m}$, under usual regularity assumptions, Broyden's method defined by Eq. (5) converges locally ${ }^{3} Q$-superlinearly [7], exactly in $2 m$ steps for linear systems, and with $R$-order at least $2^{\frac{1}{2 m}}>1$ [13].

Unfortunately, for general $K$, Eq. (5) is not a good fit. Indeed, the quadratic form $x \mapsto x^{t} x$ can be isotropic over $K^{m}$, i.e. there can be an $s_{n} \neq 0$ such that $s_{n}{ }^{t} \cdot s_{n}=0$. This is the case, for example if $s_{n}=(X, X)$ in $\mathbb{F}_{2} \llbracket X \rrbracket^{2}$. Consequently, (5) has to be modified. Trying to seek for another quadratic form that would not be isotropic is pointless, since for example there is none over $\mathbb{Q}_{p}^{m}$ for $m \geq 5$ [21].

\footnotetext{
${ }^{2} \mathrm{R}$-convergence is a weaker notion, aimed at sequences not monotonically decreasing. ${ }^{3}$ By locally, we mean that for any $x_{0}$ and $B_{0}$ in small enough balls around $x^{\star}$ and $f^{\prime}\left(x^{\star}\right)$, the following convergence property is satisfied.
} 
Remark 2.4. In the sequel, all the $B_{i}$ 's will be invertible matrices. Consequently, $s_{n+1}=0$ if and only if $f\left(x_{n}\right)=0$. We therefore adopt the convention that if for some $x_{n}$, we have $f\left(x_{n}\right)=0$, then the sequences $\left(x_{v}\right)_{v \geq n}$ and $\left(B_{v}\right)_{v \geq n}$ will be constant, and this case does not require any further development.

\section{NON-ARCHIMEDEAN ADAPTATION}

\subsection{Norms}

We use the following natural (non-normalized) norm on $K$ defined from its valuation: for any $x \in K,\|x\|=2^{-\operatorname{val}(x)}$, except for $K=$ $\mathbb{Q}_{p}$, where we take the more natural $p^{-\operatorname{val}(x)}$ over $\mathbb{Q}_{p}$. Our norm ${ }^{4}$ on $K$ can naturally be extended to $K^{m}$ : for any $x=\left(x_{1}, \ldots, x_{m}\right) \in K^{m}$, $\|x\|=\max _{i}\left|x_{i}\right|$. We denote by $\operatorname{val}(x)$ the minimal valuation among the $\operatorname{val}\left(x_{i}\right)^{\prime}$ s. It defines the norm of $x$.

Lemma 3.1. Let $\|\cdot \mid\|$ be the norm on $M_{m}(K)$ induced by $\|\cdot\|$. Let us abuse notations by denoting with $\|\cdot\|$ the max-norm on the coefficients of the matrices of $M_{m}(K)$. Then \|\|$\cdot\|\|=\|\cdot\|$.

Proof. Let $A \in M_{n}(K)$. If $x \in K^{m}$ is such that $\|x\| \leq 1$, then by ultrametricity, it is clear that $\|A x\| \leq\|A\|$, hence $\|A\|\|\leq\| A \|$. If $i \in \mathbb{N}$ is such that $\|A\|$ is obtained with a coefficient on the column of index $i$, then $\left\|A e_{i}\right\|=\|A\|$, whence the equality.

Consequently, the max-norm on the coefficients of a matrix is a matrix norm. For rank-one matrices, the computation of the norm can be made easy using the following corollary of Lemma 3.1.

Corollary 3.2. Let $a, b \in K^{m}$ be two vectors. Then

$$
\left\|a^{t} \cdot b\right\|=\|a\| \cdot\|b\| \text {. }
$$

\subsection{Constraints and optimality}

For the sequence $\left(x_{n}\right)_{n \in \mathbb{N}}$ to be well defined, the sequence $\left(u_{n}\right)_{n \in \mathbb{N}}$ must satisfy Eqs (3)-(4) and also:

$$
s_{n}{ }^{t} B_{n}^{-1} y_{n} \neq 0,
$$

to ensure Eq. (6) makes sense. Many different $u_{n}$ 's can satisfy those conditions. Over $\mathbb{R}$, Broyden's choice of $u_{n}$ defined by (5) can be characterized by minimizing the Frobenius norm of $B_{n+1}-B_{n}$. We can proceed similarly over $K$.

Lemma 3.3. If $B_{n+1}$ satisfies (2), then:

$$
\left\|B_{n+1}-B_{n}\right\| \geq \frac{\left\|y_{n}-B_{n} s_{n}\right\|}{\left\|s_{n}\right\|} .
$$

Proof. It is clear as in this case, $\left(B_{n+1}-B_{n}\right) s_{n}=y_{n}-B_{n} s_{n}$.

This inequality can become an equality with a suitable choice of $u_{n}$ as shown in the following lemma.

Lemma 3.4. Let $l$ be such that $\operatorname{val}\left(s_{n, l}\right)=\operatorname{val}\left(s_{n}\right)$. Then

$$
u_{n}=s_{n, l}^{-1} e_{l}
$$

satisfies (4) and reaches the bound in (11).

Nevertheless, this is not enough to have $B_{n}$ invertible in general, as we can see from the Sherman-Morrison formula (8):

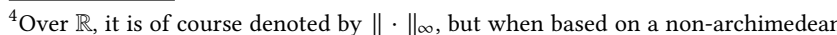
absolute value, this notation is not used since it is implicitly unambiguous: other norms such as the $\|\cdot\|_{p}$ are mostly useless.
}

LEMMA 3.5. $B_{n}$ defined by Eq.(3) is invertible if and only if

$$
u_{n-1}^{t} B_{n-1}^{-1} y_{n-1} \neq 0 \text {. }
$$

The next lemma shows how to choose $l$, up to the condition $\left(B_{n-1}^{-1} y_{n-1}\right)_{l} \neq 0$, which actually never occurs after Corollary 4.3.

Lemma 3.6. Let $l$ be the smallest index such that $\operatorname{val}\left(s_{n, l}\right)=$ $\operatorname{val}\left(s_{n}\right)$. If $\left(B_{n-1}^{-1} y_{n-1}\right), \neq 0$, then

$$
u_{n}=s_{n, l}^{-1} e_{l}
$$

satisfies Eq. (4), reaches the bound in Eq. (11) and satisfies Eq.(12).

\section{LOCAL CONVERGENCE}

\subsection{Local Linear convergence}

Let $E$ and $F$ be two finite-dimensional normed vector spaces over $K$ We denote by $L(E, F)$ the space of $K$-linear mappings from $E$ to $F$.

Definition 4.1. Let $U$ be an open subset of E. A function $f$ : $U \rightarrow F$ is strictly differentiable at $x \in U$ if there exists an $f^{\prime}(x) \in$ $L(E, F)$ satisfying the following property: for all $\varepsilon>0$, there exists a neighborhood $U_{x, \varepsilon} \subset U$ of $x$, on which for any $y, z \in U_{x, \epsilon}$ :

$$
\left\|f(z)-f(y)-f^{\prime}(x) \cdot(z-y)\right\|_{F} \leq \varepsilon \cdot\|z-y\|_{E} .
$$

Note that both $z$ and $y$ can vary. This property is natural in the ultrametric context (see 3.1.3 of [9]), as the counterpart of Fréchet differentiability over $\mathbb{R}$ does not provide meaningful local information. Polynomials and converging power series satisfy strict differentiability everywhere they are defined.

We can then adapt Theorem 3.2 of [7] in our ultrametric setting.

Theorem 4.2. Let $f: K^{m} \rightarrow K^{m}$ and $x^{\star} \in U$ be such that $f$ is strictly differentiable at $x^{\star}, f^{\prime}\left(x^{\star}\right)$ is invertible and $f\left(x^{\star}\right)=0$. Then any quasi-Newton method whose choice of $u_{n}$ yields for all $n,\left\|u_{n}\right\|=\left\|s_{n}\right\|^{-1}$ (which includes Broyden's choice of Eq. (13)), is locally $Q$-linearly converging to $x^{\star}$ with ratio $r$ for any $r \in(0,1)$.

Proof. Let $r \in(0,1)$. Let the constants $\gamma, \delta$, and $\lambda$ be satisfying: $\gamma \geq\left\|f^{\prime}\left(x^{\star}\right)^{-1}\right\|, \quad 0<\delta \leq \frac{r}{\gamma(1+r)(3-r)}, \quad 0<\lambda \leq \delta(1-r)$.

Let $\eta>0$ be given by the strict differentiability at $x^{\star}$ and such that on the ball $B\left(x^{\star}, \eta\right)$,

$$
\left\|f(z)-f(y)-f^{\prime}\left(x^{\star}\right) \cdot(z-y)\right\| \leq \lambda \cdot\|z-y\| .
$$

We restrict further $\eta$ so as to have: $\eta \leq \delta(1-r)$. Let us assume that

$$
\left\|B_{0}-f^{\prime}\left(x^{\star}\right)\right\| \leq \delta, \quad\left\|x_{0}-x^{\star}\right\|<\eta .
$$

We have from the condition on $\delta$ that $\delta \gamma(1+r)(3-r) \leq r$. Since $3-r>2$, then $2 \delta \gamma(1+r) \leq r$. Consequently,

$$
\frac{1}{1-2 \delta \gamma} \leq 1+r
$$

the denominator being non zero because $\delta<(2 \gamma)^{-1}$.

Since $\left\|f^{\prime}\left(x^{\star}\right)^{-1}\right\| \leq \gamma$ and $\left\|B_{0}-f^{\prime}\left(x^{\star}\right)\right\|<2 \delta$, the Banach Perturbation Lemma ([20] page 45) in the Banach algebra $M_{m}(K)$ implies that $B_{0}$ is invertible and:

$$
\left\|B_{0}^{-1}\right\| \leq \frac{\gamma}{1-2 \gamma \delta} \leq(1+r) \gamma .
$$

We can now estimate what happens to $x_{1}=x_{0}-B_{0}^{-1} f\left(x_{0}\right)$. 


$$
\begin{aligned}
\left\|x_{1}-x^{\star}\right\|= & \left\|x_{0}-x^{\star}-B_{0}^{-1} f\left(x_{0}\right)\right\|, \\
= & \|-B_{0}^{-1}\left(f\left(x_{0}\right)-f\left(x^{\star}\right)-f^{\prime}\left(x^{\star}\right) \cdot\left(x_{0}-x^{\star}\right)\right) \\
& -B_{0}^{-1}\left(f^{\prime}\left(x^{\star}\right)\left(x_{0}-x^{\star}\right)-B_{0}\left(x_{0}-x^{\star}\right)\right) \|, \\
= & \|-B_{0}^{-1}\left(f\left(x_{0}\right)-f\left(x^{\star}\right)-f^{\prime}\left(x^{\star}\right) \cdot\left(x_{0}-x^{\star}\right)\right) \\
& -B_{0}^{-1}\left(\left(f^{\prime}\left(x^{\star}\right)-B_{0}\right)\left(x_{0}-x^{\star}\right)\right) \|, \\
\leq & \left\|B_{0}^{-1}\right\|\left(\lambda\left\|x_{0}-x^{\star}\right\|+2 \delta\left\|x_{0}-x^{\star}\right\|\right), \\
\leq & \left\|B_{0}^{-1}\right\|(\lambda+2 \delta)\left\|x_{0}-x^{\star}\right\|, \\
\leq & \gamma(1+r)(\delta(1-r)+2 \delta)\left\|x_{0}-x^{\star}\right\|, \\
\leq & \gamma(1+r) \delta(3-r)\left\|x_{0}-x^{\star}\right\| \\
\leq & r\left\|x_{0}-x^{\star}\right\| .
\end{aligned}
$$

Consequently, $\left\|x_{1}-x^{\star}\right\| \leq r\left\|x_{0}-x^{\star}\right\|$ and $\left\|x_{1}-x^{\star}\right\| \leq r \eta<\eta$, i.e. $x_{1} \in B\left(x^{\star}, \eta\right)$.

Eq. (3) defines $B_{1}$ by $B_{1}=B_{0}-\left(y_{1}-B_{0} s_{1}\right) \cdot u_{1}^{t}$ for some $u_{1}$ verifying $\left\|u_{1}\right\|=\left\|s_{1}\right\|^{-1}$ (see Eqs. (4), Corollary 3.2). Then:

$$
\left\|B_{1}-B_{0}\right\|=\left\|f\left(x_{1}\right)-f\left(x_{0}\right)-B_{0}\left(x_{1}-x_{0}\right)\right\| \cdot\left\|x_{1}-x_{0}\right\|^{-1} \text {. }
$$

Therefore,

$$
\begin{aligned}
\left\|B_{1}-f^{\prime}\left(x^{\star}\right)\right\| \leq & \max \left(\left\|B_{0}-f^{\prime}\left(x^{\star}\right)\right\|,\right. \\
& \left.\left\|f\left(x_{1}\right)-f\left(x_{0}\right)-B_{0}\left(x_{1}-x_{0}\right)\right\|\left\|x_{1}-x_{0}\right\|^{-1}\right), \\
\leq & \max \left(\left\|B_{0}-f^{\prime}\left(x^{\star}\right)\right\|,\right. \\
& \left\|\left(B_{0}-f^{\prime}\left(x^{\star}\right)\right)\left(x_{1}-x_{0}\right)\right\|\left\|x_{1}-x_{0}\right\|^{-1}, \\
& \left.\left\|f\left(x_{1}\right)-f\left(x_{0}\right)-f^{\prime}\left(x^{\star}\right)\left(x_{1}-x_{0}\right)\right\|\left\|x_{1}-x_{0}\right\|^{-1}\right), \\
\leq & \max (\delta, \lambda) \leq \delta .
\end{aligned}
$$

We can then carry on and prove by induction that for all $k$,

(i) $\left\|x_{k}-x^{\star}\right\| \leq r^{k}\left\|x_{0}-x^{\star}\right\|, \quad$ and $\quad$ (ii) $B_{k} \in B\left(f^{\prime}\left(x^{\star}\right), \delta\right)$. (1

Heredity for Inequality (19)-(i) comes from: a same use of the Banach Perturbation Lemma on $B_{k}$ so that $B_{k}$ is invertible; that $\left\|B_{k}^{-1}\right\| \leq(1+r) \gamma$ and by repeating the computations (16) to (17):

$$
\begin{aligned}
\left\|x_{k+1}-x^{\star}\right\| & \leq\left\|B_{k}\right\|^{-1}(\lambda+2 \delta)\left\|x_{k}-x^{\star}\right\|, \\
& \leq(1+r) \gamma \delta(3-r)\left\|x_{k}-x^{\star}\right\|, \\
& \leq r\left\|x_{k}-x^{\star}\right\| .
\end{aligned}
$$

We can deal with (19)-(ii) using a similar computation as (18):

$\left\|B_{k+1}-f^{\prime}\left(x^{\star}\right)\right\| \leq \max \left(\left\|B_{k}-f^{\prime}\left(x^{\star}\right)\right\|\right.$,

$$
\begin{aligned}
& \left.\left\|f\left(x_{k+1}\right)-f\left(x_{k}\right)-B_{k}\left(x_{k+1}-x_{k}\right)\right\|\left\|x_{k+1}-x_{k}\right\|^{-1}\right) \\
\leq & \max \left(\left\|B_{k}-f^{\prime}\left(x^{\star}\right)\right\|,\right. \\
& \left.\left\|f\left(x_{k+1}\right)-f\left(x_{k}\right)-f^{\prime}\left(x^{\star}\right)\left(x_{k+1}-x_{k}\right)\right\|\left\|x_{k+1}-x_{k}\right\|^{-1}\right), \\
\leq & \max (\delta, \lambda) \leq \delta .
\end{aligned}
$$

Corollary 4.3. Locally, one can take definition (13) to define all the $u_{n}$ 's and all the $B_{n}$ 's will still be invertible.

Proof. With the assumptions of the proof of Theorem 4.2, for $u_{n}$ defined by (13), $\left\|u_{n-1}\right\|=\left\|s_{n-1}\right\|^{-1}$ and (4) are satisfied, and by the Banach Perturbation Lemma, $B_{n}$ defined by (3) is invertible.

Remark 4.4. The fact that Broyden's method has locally Q-linear convergence with ratio $r$ for any $r$ is not enough to prove that ithas Q-superlinear convergence. Indeed, as $x_{k}$ is going closer to $x^{\star}$, there is no reason for $B_{k}$ to get closer to $f^{\prime}\left(x^{\star}\right)$. Consequently, we cannot expect from the previous result that $x_{k}$ and $B_{k}$ enter loci of smaller ratio of convergence as $k$ goes to infinity. In fact, in general, $B_{k}$ does not converge to $f^{\prime}\left(x^{\star}\right)$.

Finally, the next lemma, consequence of the previous theorem, will be useful in the next subsection to obtain the R-superlinear convergence.

LEMma 4.5. Using the same notations as in the proof of Theorem 4.2, if $r \leq\left(\frac{\gamma\left\|f^{\prime}\left(x^{\star}\right)\right\|}{2}\right)^{-1}$, and $\left\|B_{0}-f^{\prime}\left(x^{\star}\right)\right\|<\delta$ and $\left\|x_{0}-x^{\star}\right\|<\eta$, then for all $n \in \mathbb{N}$,

$$
\left\|f_{n+1}\right\| \leq\left\|f_{n}\right\|
$$

Proof. Let $n \in \mathbb{N}$. We have $\left\|s_{n}\right\| \leq r\left\|s_{n-1}\right\|$. Indeed, from $\left\|x_{n+1}-x_{n}\right\| \leq \max \left(\left\|x_{n+1}-x^{\star}\right\|,\left\|x^{\star}-x_{n}\right\|\right)$, and $\left\|x_{n+1}-x_{n}\right\|<$ $\left\|x_{n}-x^{\star}\right\|$, we see that $\left\|s_{n}\right\|=\left\|x^{\star}-x_{n}\right\| \leq r\left\|x^{\star}-x_{n-1}\right\|=r\left\|s_{n-1}\right\|$.

Then using $(\mathrm{QN})$ and the Q-linear convergence with ratio $r$, we get that $\left\|f_{n+1}\right\| \leq r\left\|B_{n+1}\right\|\left\|B_{n}^{-1}\right\|\left\|f_{n}\right\|$. Using (20), the definition of $\delta, \gamma$ in (15), and the fact that $0<r<1$, we get that $\left\|B_{n+1}\right\|\left\|B_{n}^{-1}\right\| \leq$ $2 \gamma\left\|f^{\prime}\left(x^{\star}\right)\right\|$, which concludes the proof.

\subsection{Local R-superlinear convergence}

We first remark that the $2 n$-step convergence in the linear case proved by Gay in [13] is still valid. Indeed, it is only a matter of linear algebra.

Theorem 4.6 (Theorem 2.2 in [13]). If $f$ is defined by $f(x)=$ $A x-b$ for some $A \in G L_{m}(K)$, then any quasi-Newton method converges in at most $2 m$ steps (i.e. $f\left(x_{2 m}\right)=0$ ).

With this and under a stronger differentiability assumption on $f$, we can obtain R-superlinearity, similarly to Theorem 3.1 of [13]. The proof also follows the main steps thereof.

THeOREM 4.7. Let us assume that on a neighborhood $U$ of $x^{\star}$, there is a $c_{0} \in \mathbb{R}_{>0}$ such that $f$ satisfies ${ }^{5}$

$$
\forall x, y \in U,\left\|f(x)-f(y)-f^{\prime}\left(x^{\star}\right) \cdot(x-y)\right\| \leq c_{0}\|x-y\|^{2} .
$$

Then there are $\eta, \delta$ and $\Gamma$ in $\mathbb{R}_{>0}$ such that if $x_{0} \in B\left(x^{\star}, \eta\right)$ and $B_{0} \in B\left(f^{\prime}\left(x^{\star}\right), \delta\right)$, then for any $w \in \mathbb{Z}_{\geq 0}$,

$$
\left\|x_{w+2 m}-x^{\star}\right\| \leq \Gamma\left\|x_{w}-x^{\star}\right\|^{2} .
$$

Proof. Step 1: Preliminaries. Condition (21) is stronger than strict differentiability as stated in Theorem 4.2. From its proof and Lemma 4.5, let $r \in(0,1)$ and $\gamma \geq\left\|f^{\prime}\left(x^{\star}\right)^{-1}\right\|$, as well as $\eta$ and $\delta$ such that: $r \leq\left(\frac{\gamma\left\|f^{\prime}\left(x^{\star}\right)\right\|}{2}\right)^{-1}$, and if $x_{0} \in B\left(x^{\star}, \eta\right)$ and $B_{0} \in B\left(f^{\prime}\left(x^{\star}\right), \delta\right)$, the sequences $\left(x_{n}\right)_{n \in \mathbb{N}}$ and $\left(B_{n}\right)_{n \in \mathbb{N}}$ defined by Broyden's method (using (13)) are well defined and moreover the four following inequalities are satisfied: for any $k \in \mathbb{N}$,

$$
\begin{array}{ll}
\left\|B_{k}-f^{\prime}\left(x^{\star}\right)\right\| \leq \delta, & \left\|x_{k+1}-x^{\star}\right\| \leq r\left\|x_{k}-x^{\star}\right\|, \\
\left\|B_{k}^{-1}\right\| \leq(1+r) \gamma, & \left\|f\left(x_{k+1}\right)\right\| \leq\left\|f\left(x_{k}\right)\right\| .
\end{array}
$$

Let $x_{0} \in B\left(x^{\star}, \eta\right), B_{0} \in B\left(f^{\prime}\left(x^{\star}\right), \delta\right)$, and $\left(x_{n}\right)_{n \in \mathbb{N}}$ and $\left(B_{n}\right)_{n \in \mathbb{N}}$ be defined by Broyden's method. Let $w \in \mathbb{N}$ and $h=\left\|x_{w}-x^{\star}\right\|$. We

\footnotetext{
${ }^{5}$ This condition is satisfied by polynomials or converging power series.
} 
must show that there is a $\Gamma$, independent of $w$ such that $\| x_{w+2 m}-$ $x^{\star} \| \leq \Gamma h^{2}$.

Step 2: reference to a linear map. Let the linear affine map $\hat{f}(x)=$ $f^{\prime}\left(x^{\star}\right)\left(x-x^{\star}\right)$, and $\hat{x_{0}}=x_{w}$ and $\hat{B}_{0}=B_{w}$. Broyden's method (using first (13)) applied to those data produces the sequences $\left(\hat{x}_{n}\right)_{n \in \mathbb{N}}$ and $\left(\hat{B}_{n}\right)_{n \in \mathbb{N}}$, which are constant for $n \geq 2 m$, as a result of Theorem 4.2. We define similarly $\hat{s}_{n}=\hat{x}_{n+1}-\hat{x}_{n}$. We have again for all $k \in \mathbb{N}$ the four inequalities:

$$
\begin{array}{ll}
\left\|\hat{B}_{k}-f^{\prime}\left(x^{\star}\right)\right\| \leq \delta, & \left\|\hat{x}_{k+1}-x^{\star}\right\| \leq r\left\|\hat{x}_{k}-x^{\star}\right\|, \\
\left\|\hat{B}_{k}^{-1}\right\| \leq(1+r) \gamma & \left\|\hat{f}\left(x_{k+1}\right)\right\| \leq\left\|\hat{f}\left(x_{k}\right)\right\| .
\end{array}
$$

The key to the proof is that $\hat{x}_{2 m}=x^{\star}$ and $\hat{x}_{k}$ and $x_{w+k}$ are not too much far apart.

Step 3: Statement of the induction. More concretely, we prove by induction on $j$ that there exist $\gamma_{1, j}$ and $\gamma_{2, j}$, independent of $w$, such that for $0 \leq j \leq 2 m$, we have the two inequalities:

$$
\begin{gathered}
\left\|B_{w+j}-\hat{B}_{j}\right\| \cdot\left\|f_{w+j}\right\| \leq \gamma_{1, j} h^{2}, \\
\left\|x_{w+j}-\hat{x}_{j}\right\| \leq \gamma_{2, j} h^{2} .
\end{gathered}
$$

Step 4: Base case. Since $B_{w}=\hat{B}_{0}$ and $x_{w}=\hat{x}_{0},\left(E_{1,0}\right)$ and $\left(E_{2,0}\right)$ are clear, with $\gamma_{1,0}=\gamma_{2,0}=0$. Now, let us assume that $\left(E_{1, k}\right)$ and $\left(E_{2, k}\right)$ are true for a given $k$ such that $0 \leq k<2 m$.

Step 5: We first prove $\left(E_{2, k+1}\right)$. One part of the inequality (22) is obtained thanks to: $B_{w+k}^{-1}-\hat{B}_{k}^{-1}=B_{w+k}^{-1}\left(\hat{B}_{k}-B_{w+k}\right) \hat{B}_{k}^{-1}$.

$$
\begin{aligned}
& \left\|s_{w+k}-\hat{s}_{k}\right\|=\left\|B_{w+k}^{-1} f_{w+k}-\hat{B}_{k}^{-1} \hat{f}\left(\hat{x}_{k}\right)\right\| \\
& \leq \max \left(\left\|B_{w+k}^{-1}\right\| \cdot\left\|\hat{B}_{k}^{-1}\right\| \cdot\left\|B_{w+k}-\hat{B}_{k}\right\| \cdot\left\|f_{w+k}\right\|,\right. \\
& \left.\quad\left\|\hat{B}_{k}^{-1}\right\| \cdot\left\|f_{w+k}-\hat{f}\left(\hat{x}_{k}\right)\right\|\right) \\
& \leq\left\|\hat{B}_{k}^{-1}\right\| \max \left(\left\|B_{w+k}^{-1}\right\| \cdot\left\|B_{w+k}-\hat{B}_{k}\right\| \cdot\left\|f_{w+k}\right\|,\right. \\
& \left.\quad\left\|f_{w+k}-\hat{f}\left(x_{w+k}\right)\right\|,\left\|\hat{f}\left(x_{w+k}\right)-\hat{f}\left(\hat{x}_{k}\right)\right\|\right)
\end{aligned}
$$

The first term on the r.h.s. of (23) is upper-bounded by $(1+r)^{2} \gamma^{2} \gamma_{1, k} h^{2}$ using $\left(E_{1, k}\right)$ and $\left\|B_{w+k}^{-1}\right\| \leq(1+r) \gamma$.

For the second term of (23), using (21):

$$
\left\|f_{w+k}-f\left(x^{\star}\right)-f^{\prime}\left(x^{\star}\right) \cdot\left(x_{w+k}-x^{\star}\right)\right\| \leq c_{0}\left\|x_{w+k}-x^{\star}\right\|^{2}
$$

and $\left\|x_{w+k}-x^{\star}\right\| \leq\left\|x_{w}-x^{\star}\right\|=h$, it is upper-bounded by $c_{0} h^{2}$. Finally, the last term is equal to $f^{\prime}\left(x^{\star}\right)\left(x_{w+k}-\hat{x}_{k}\right)$ whose norm is upper-bounded by $\left\|f^{\prime}\left(x^{\star}\right)\right\| \gamma_{2, k} h^{2}$ thanks to $\left(E_{2, k}\right)$. This is enough to define $\gamma_{3, k}$ such that $\left\|s_{w+k}-\hat{s}_{k}\right\| \leq \gamma_{3, k} h^{2} \quad$ (†). Consequently, with $\gamma_{2, k+1}=\max \left(\gamma_{3, k}, \gamma_{2, k}\right)$, we do have $\left\|x_{w+k+1}-\hat{x}_{k+1}\right\| \leq$ $\gamma_{2, k+1} h^{2}$, and $\left(E_{2, k+1}\right)$ is satisfied.

Step 6.0: We now prove $\left(E_{1, k+1}\right)$. We first deal with some preliminary cases. If $s_{w+k}=0$, (that is $\left.x_{w+k+1}=x_{w+k}\right)$ then the property (2) $s_{w+k}=-B_{w+k}^{-1} f_{w+k}$ implies that $f_{w+k}=0$, and the property $B_{w+k+1} s_{w+k}=y_{w+k}$ implies that $f_{w+k}=f_{w+k+1}=0$ Thus $\left(E_{1, k+1}\right)$ is satisfied with $\gamma_{1, k+1}=0$. If $\hat{s}_{k}=0$, then similarly $\hat{f}\left(\hat{x}_{w+k}\right)=\hat{f}\left(\hat{x}_{w+k+1}\right)=0$. Therefore, as we have seen before,

$$
\begin{aligned}
\left\|f_{w+k+1}\right\| & =\left\|f_{w+k+1}-\hat{f}\left(x_{w+k+1}\right)+\hat{f}\left(x_{w+k+1}\right)-\hat{f}\left(\hat{x}_{k+1}\right)\right\|, \\
& \leq \max \left(c_{0},\left\|f^{\prime}\left(x^{\star}\right)\right\| \gamma_{2, k+1}\right) h^{2} .
\end{aligned}
$$

Then, using that $\left\|B_{w+k+1}-\hat{B}_{k+1}\right\| \leq \max \left(\left\|B_{w+k+1}-f^{\prime}\left(x^{\star}\right)\right\|, \| \hat{B}_{k+1}-\right.$ $\left.f^{\prime}\left(x^{\star}\right) \|\right) \leq \delta,\left(E_{1, k+1}\right)$ is satisfied with:

$$
\gamma_{1, k+1}=\delta h^{2} \max \left(c_{0},\left\|f^{\prime}\left(x^{\star}\right)\right\| \gamma_{2, k+1}\right) \text {. }
$$

Step 6.1 : We can now assume that both $s_{k}$ and $\hat{s}_{k}$ are non zero. To prove that there is a $\gamma_{1, k+1}$ (independent of $w$ ) such that $\left(E_{1, k+1}\right)$ holds, then in view of the fact that $\left\|f_{w+k+1}\right\| \leq\left\|f_{w+k}\right\|$ (Lemma 4.5) of $\left(E_{1, k}\right)$ and of the definition (Eq. (3)) of $B_{k+1}$ and $\hat{B}_{k+1}$, it is enough to prove that there is some $\gamma_{4, k+1}$ (independent of $w$ ) such that:

$$
\begin{aligned}
& \|\left(y_{w+k}-B_{w+k} s_{w+k}\right) u_{w+k}{ }^{t}- \\
& \quad\left(\hat{y}_{k}-\hat{B}_{k} \hat{s}_{k}\right) \hat{u}_{k}^{t}\|\cdot\| f_{w+k+1} \| \leq \gamma_{4, k+1} h^{2} .
\end{aligned}
$$

Using that $\left\|f_{w+k+1}\right\| \leq\left\|f_{w+k}\right\|$ (by Lemma 4.5), we obtain:

$$
\begin{aligned}
\left\|f_{w+k+1}\right\| \cdot & \left\|\left(y_{w+k}-B_{w+k} s_{w+k}\right) u_{w+k}^{t}-\left(\hat{y}_{k}-\hat{B}_{k} \hat{s}_{k}\right) \hat{u}_{k}^{t}\right\| \\
\leq & \left\|f_{w+k}\right\| \max \left(\left\|y_{w+k}-f^{\prime}\left(x^{\star}\right) s_{w+k}\right\| \cdot\left\|u_{w+k}{ }^{t}\right\|,\right. \\
& \left.\left\|\left(f^{\prime}\left(x^{\star}\right)-B_{w+k}\right) s_{w+k} u_{w+k}^{t}-\left(f^{\prime}\left(x^{\star}\right)-\hat{B}_{k}\right) \hat{s}_{k} \hat{u}_{k}^{t}\right\|\right) \\
\leq & \left\|f_{w+k}\right\| \max \left(\left\|y_{w+k}-f^{\prime}\left(x^{\star}\right) s_{w+k}\right\| \cdot\left\|u_{w+k}{ }^{t}\right\|,\right. \\
& \left\|\left(f^{\prime}\left(x^{\star}\right)-\hat{B}_{k}\right)\left(s_{w+k} u_{w+k}^{t}-\hat{s}_{k} \hat{u}_{k}^{t}\right)\right\|, \\
& \left.\left\|\left(B_{w+k}-\hat{B}_{k}\right) s_{w+k} u_{w+k}{ }^{t}\right\|\right) .
\end{aligned}
$$

Step 6.2: From $f_{w+k}=-B_{w+k} s_{w+k}$, we have $\left\|f_{w+k}\right\| \leq\left\|s_{w+k}\right\|$. $\max \left(\left\|B_{w+k}-f^{\prime}\left(x^{\star}\right)\right\|,\left\|f^{\prime}\left(x^{\star}\right)\right\|\right) \leq\left\|s_{w+k}\right\| \cdot \max \left(\delta,\left\|f^{\prime}\left(x^{\star}\right)\right\|\right)(\bullet)$. Otoh by (21), $\left\|y_{w+k}-f^{\prime}\left(x^{\star}\right) s_{w+k}\right\| \leq c_{0}\left\|s_{w+k}\right\|^{2}$. It follows that the first term (25) can be upper-bounded in the following way:

$(25) \leq c_{0}\left\|s_{w+k}\right\|^{3}\left\|u_{w+k}{ }^{t}\right\| \max \left(\delta,\left\|f^{\prime}\left(x^{\star}\right)\right\|\right) \leq c_{0} h^{2} \max \left(\delta,\left\|f^{\prime}\left(x^{\star}\right)\right\|\right)$, the rightmost inequality being obtained from $\left\|u_{w+k}{ }^{t}\right\|=\left\|s_{w+k}\right\|^{-1}$ and $\left\|s_{w+k}\right\| \leq \max \left(\left\|x_{w+k+1}-x^{\star}\right\|,\left\|x_{w+k}-x^{\star}\right\|\right)=\| x_{w+k}-$ $x^{\star}\|\leq\| x_{w}-x^{\star} \|=h$.

Step 6.3: The third one (27) can be upper-bounded using $\left(E_{1, k}\right)$ :

$$
\text { (27) } \leq\left\|f_{w+k}\right\|\left\|\left(B_{w+k}-\hat{B}_{k}\right) s_{w+k} u_{w+k}^{t}\right\| \leq \gamma_{1, k} h^{2} .
$$

Step 6.4: For the second one (26), observe that:

$$
s_{w+k} u_{w+k}^{t}-\hat{s}_{k} \hat{u}_{k}^{t}=\left(s_{w+k}-\hat{s}_{k}\right) u_{w+k}^{t}-\hat{s}_{k}\left(u_{w+k}^{t}-\hat{u}_{k}^{t}\right) \text {. }
$$

The first term is easy to manage using the previous inequality (•) on $\left\|f_{w+k}\right\|$, the inequality (†) on $\left\|s_{w+k}-\hat{s}_{k}\right\|$ and $\left\|s_{w+k}\right\|\left\|u_{w+k} t^{t}\right\|=1$ :

$$
\left\|f_{w+k}\right\| \cdot\left\|\left(s_{w+k}-\hat{s}_{k}\right) u_{w+k}{ }^{t}\right\| \leq \max \left(\delta,\left\|f^{\prime}\left(x^{\star}\right)\right\|\right) \gamma_{3, k} h^{2} .
$$

The second one of Eq. (28) is a little bit trickier. Define as in (13), $u_{w+k}=s_{w+k, l}^{-1} e_{l}$ and $\hat{u}_{k}=\hat{s}_{k, \hat{l}}^{-1} e_{\hat{l}}$ for some given $l$ and $\hat{l}$.

If $l=\hat{l}$, we have: (the last inequality below follows from ( $\$)$ ).

$$
\begin{aligned}
\left\|u_{w+k}-\hat{u}_{k}\right\| & =\left|s_{w+k, l}^{-1}-\hat{s}_{k, l}^{-1}\right|=\frac{\left|s_{w+k, l}-\hat{s}_{k, l}\right|}{\left|s_{w+k, l}\right| \cdot\left|\hat{s}_{k, l}\right|}=\frac{\left|s_{w+k, l}-\hat{s}_{k, l}\right|}{\left\|s_{w+k}\right\| \cdot\left\|\hat{s}_{k}\right\|} \\
& \leq \frac{\left\|s_{w+k}-\hat{s}_{k}\right\|}{\left\|s_{w+k}\right\| \cdot\left\|\hat{s}_{k}\right\|} \leq \frac{\gamma_{3, k} h^{2}}{\left\|s_{w+k}\right\| \cdot\left\|\hat{s}_{k}\right\|} .
\end{aligned}
$$

From this and from $\left\|f_{w+k}\right\|=\left\|B_{w+k}\right\| \cdot\left\|s_{w+k}\right\|$ we get:

$$
\left\|f_{w+k}\right\| \cdot\left\|u_{w+k}-\hat{u}_{k}\right\| \cdot\left\|\hat{s}_{k}\right\| \leq \gamma_{3, k} \max \left(\delta,\left\|f^{\prime}\left(x^{\star}\right)\right\|\right) h^{2} .
$$

If $l \neq \hat{l}$, then either $\left\|s_{w+k}-\hat{s}_{k}\right\|=\left\|s_{w+k}\right\|$, if $\left\|\hat{s}_{k}\right\| \leq\left\|s_{w+k}\right\|$, or $\left\|s_{w+k}-\hat{s}_{k}\right\|=\left\|\hat{s}_{k}\right\|$, if $\left\|s_{w+k}\right\| \leq\left\|\hat{s}_{k}\right\|$. In the first case, we have

$$
\left\|u_{w+k}-\hat{u}_{k}\right\|=\left\|\hat{s}_{k}\right\|^{-1} \text {, }
$$


and then, the second term of (28) multiplied by $\left\|f_{w+k}\right\|$ verifies:

$$
\begin{aligned}
\left\|f_{w+k}\right\| \cdot\left\|u_{w+k}-\hat{u}_{k}\right\| \cdot\left\|\hat{s}_{k}\right\| & \leq \max \left(\delta,\left\|f^{\prime}\left(x^{\star}\right)\right\|\right)\left\|s_{w+k}\right\| \\
& \leq \max \left(\delta,\left\|f^{\prime}\left(x^{\star}\right)\right\|\right) \gamma_{3, k} h^{2} .
\end{aligned}
$$

The second case follows with the same computation. Eqs (31) (30) (29) prove together the bound on the expression (26) in (28). In turn with the bounds on the terms (25) and (27), prove (24). This concludes the proof of $\left(E_{1, k+1}\right)$, and finally the induction.

Step 7: Consequently, $\left\|x_{w+2 m}-\hat{x}_{2 m}\right\| \leq \gamma_{2,2 m} h^{2}$. Thanks to Theorem 4.2, $\hat{x}_{2 m}=x^{\star}$, and thus, we have proved that for any $w$,

$$
\left\|x_{w+2 m}-x^{\star}\right\| \leq \gamma_{2,2 m}\left\|x_{w}-x^{\star}\right\|^{2} .
$$

Theorem 4.7 has for immediate consequence:

TheOREM 4.8. Broyden's method has locally R-order of convergence $2^{\frac{1}{2 m}}$.

Proof. Let us take $x_{0}$ and $B_{0}$ as in the proof of the previous theorem, and same constants and notations. For any $w, \| x_{w+2 m}-$ $x^{\star}\|\leq \Gamma\| x_{w}-x^{\star} \|^{2}$.

Consequently, for $0 \leq k<2 m, l \in \mathbb{N}$, and $\mu=2^{1 / 2 m}$,

$$
\begin{aligned}
\left\|x_{2 l m+k}-x^{\star}\right\|^{\mu^{-2 l m-k}} & \leq\left\|x_{k}-x^{\star}\right\|^{2^{l} \mu^{-2 l m-k}} \Gamma^{\left(2^{l}-1\right) \mu^{-2 l m-k}} \\
& \leq\left\|x_{k}-x^{\star}\right\|^{2^{l} 2^{-l-\frac{k}{2 m}}} \Gamma^{\left(2^{l}-1\right) 2^{-l-\frac{k}{2 m}}} \\
& \leq\left\|x_{k}-x^{\star}\right\|^{2^{-\frac{k}{2 m}}} \Gamma^{\left(1-2^{-l}\right) 2^{-\frac{k}{2 m}}} .
\end{aligned}
$$

For simplicity, we can assume that $\Gamma \geq 1$. Thus,

$$
\begin{aligned}
\left\|x_{2 l m+k}-x^{\star}\right\|^{\mu^{-2 l m-k}} & \leq\left\|x_{k}-x^{\star}\right\|^{2^{-\frac{k}{2 m}}} \Gamma^{2^{-\frac{k}{2 m}}} . \\
& \leq\left\|x_{0}-x^{\star}\right\|^{2^{-\frac{k}{2 m}}} \Gamma^{2^{-\frac{k}{2 m}}} .
\end{aligned}
$$

Therefore, for $\left\|x_{0}-x^{\star}\right\|$ small enough, we get that for all $k$ such that $0 \leq k<2 m,\left\|x_{0}-x^{\star}\right\|^{2^{-\frac{k}{2 m}}} \Gamma^{2^{-\frac{k}{2 m}}}<1$, and hence, $\lim \sup _{s}\left\|x_{s}-x^{\star}\right\|^{\mu^{s}}<1$. From 9.2.7 of [20], we then obtain that Broyden's method do have locally R-order of convergence $2^{\frac{1}{2 m}}$.

\section{QUESTIONS ON Q-SUPERLINEARITY}

A Q-order of $\mu$ implies an R-order of $\mu$. The converse is not true. Over $\mathbb{R}$, one of the most important result concerning Broyden's method is that it is Q-superlinear. The extension of this result to the non-archimedean case remains an open question.

\subsection{Dimension 1: secant method}

In dimension one, Broyden's method reduces to the secant method.

It is known since [1] that the $p$-adic secant method applied on polynomials has order $\Phi$, the golden ratio. Its generalization to a general non-archimedean context is straightforward.

Proposition 5.1. Let us assume that $m=1$ and on a neighborhood $U$ of $x^{\star}$, there is a $c_{0} \in \mathbb{R}_{>0}$ such that $f$ satisfies (21) on $U$. Then the secant method has locally $Q$-order of convergence $\Phi$.

Proof. Let us assume that we are in the same context as in the proof of Theorem 4.7, with some Q-linear convergence of ratio $r<1$. Let us define $\varepsilon_{k}=x_{k}-x^{\star}$ for $k \in \mathbb{N}$. For all $k \in \mathbb{N}$, $\left|\varepsilon_{k+1}\right|<\left|\varepsilon_{k}\right|$. Then by ultrametricity, $\left|x_{k+1}-x_{k}\right|=\left|\varepsilon_{k}\right|$. Also, we further assume that $c_{0}\left|\varepsilon_{0}\right|<\left|f^{\prime}\left(x^{\star}\right)\right|$ so that for all $k \in \mathbb{N}$, $\left|f^{\prime}\left(x^{\star}\right) \times\left(x_{k+1}-x_{k}\right)\right|>c_{0}\left|\left(x_{k+1}-x_{k}\right)\right|^{2}$, which also implies by ultrametricity and (21) that for all $k \in \mathbb{N}$,

$$
\left|f\left(x_{k+1}\right)-f\left(x_{k}\right)\right|=\left|f^{\prime}\left(x^{\star}\right) \times\left(x_{k+1}-x_{k}\right)\right| .
$$

Similarly, $\left|f\left(x_{k}\right)\right|=\left|f^{\prime}\left(x^{\star}\right)\right|\left|\varepsilon_{k}\right|$.

Now, let $n \in \mathbb{Z}_{>0}$. Broyden's iteration is given by:

$$
x_{n+1}=x_{n}-\frac{x_{n}-x_{n-1}}{f\left(x_{n}\right)-f\left(x_{n-1}\right)} \text {. }
$$

It rewrites as:

$$
\begin{aligned}
\left|\varepsilon_{n+1}\right| & =\left|\varepsilon_{n}-\frac{\varepsilon_{n} f\left(x_{n}\right)-\varepsilon_{n-1} f\left(x_{n}\right)}{f\left(x_{n}\right)-f\left(x_{n-1}\right)}\right|=\left|\frac{\varepsilon_{n-1} f\left(x_{n}\right)-\varepsilon_{n} f\left(x_{n-1}\right)}{f\left(x_{n}\right)-f\left(x_{n-1}\right)}\right| \\
& \leq c_{0} \frac{\max \left(\left|\varepsilon_{n-1}\right|\left|\varepsilon_{n}\right|^{2},\left|\varepsilon_{n-1}\right|^{2}\left|\varepsilon_{n}\right|\right)}{\left|f\left(x_{n}\right)-f\left(x_{n-1}\right)\right|} \leq \frac{c_{0}}{\left|f^{\prime}\left(x^{\star}\right)\right|}\left|\varepsilon_{n}\right|\left|\varepsilon_{n-1}\right| .
\end{aligned}
$$

Let us write $C=\frac{c_{0}}{\left|f^{\prime}\left(x^{\star}\right)\right|}$ and $v_{n}=C \varepsilon_{n}$. Then, $v_{n+1} \leq v_{n} v_{n-1}$ for any $n>0$ and consequently,

$$
\frac{v_{n+1}}{v_{n}^{\Phi}} \leq v_{n}^{1-\Phi} v_{n-1} \leq\left(\frac{v_{n}}{v_{n-1}^{\Phi}}\right)^{1-\Phi},
$$

as $\Phi^{2}=\Phi+1$. If we define $\left(Y_{n}\right)_{n \in \mathbb{Z}_{\geq 1}}$ by $Y_{1}=\frac{v_{1}}{v_{0}^{\Phi}}$ and $Y_{n+1}=Y_{n}^{1-\Phi}$, then $\frac{v_{n+1}}{v_{n}^{\Phi}} \leq Y_{n}$. Since $|1-\Phi|<1$, then $Y_{n}$ converges to 1 . Therefore, it is bounded by some $D \in \mathbb{R}_{+}$, and $\frac{v_{n+1}}{v_{n}^{\phi}} \leq D$ for all $n \in \mathbb{Z}_{\geq 1}$. This concludes the proof.

\subsection{General case}

Over $\mathbb{R}$, Broyden's method is known to converge Q-superlinearly. The key point is that for any $E \in M_{m}(\mathbb{R})$ and $s \in \mathbb{R}^{m} \backslash\{0\}$,

$$
\left\|E\left(I-\frac{s \cdot s^{t}}{\left(s^{t} \cdot s\right)}\right)\right\|_{F}^{2}=\|E\|_{F}^{2}-\left(\frac{\|E s\|_{2}}{\|s\|_{2}}\right)^{2},
$$

equation (5.5) of [10]. The minus sign is a blessing as it allows the appearance of a telescopic sum which plays a key role in proving that $\frac{\left\|x_{n+1}-x^{\star}\right\|}{\left\|x_{n}-x^{\star}\right\|}$ converges to zero. Unfortunately, there does not seem to be a non-archimedean analogue to this equality. Thanks to Theorem 4.7, we nevertheless believe in the following conjecture.

Conjecture 5.2. In the same setting as Theorem 4.7, Broyden's method has locally $Q$-superlinear convergence.

\section{FINITE PRECISION}

\subsection{Design and notations}

One remarkable feature of Newton's method in an ultrametric context is the way it can handle precision. For example, if $\pi$ is a uniformizer, if we assume that $\left\|f^{\prime}\left(x^{\star}\right)^{-1}\right\|=1, x_{n}$ known at precision $O\left(\pi^{2^{n}}\right)$ is enough to obtain $x_{n+1}$ at precision $O\left(\pi^{2^{n+1}}\right)$. To that intent, it thus suffices to double the precision at each new iteration. Hence the working precision of Newton's method can be taken to grow at the same rate as the rate of convergence.

The handling of precision is more subtle in Broyden. This is however crucial to design efficient implementations. Note that in the real numerical setting, most works using Broyden's methods are employing fixed finite precision arithmetic, and do not address 
precision. Additionally, the lack of a knowledge of a precise exponent of convergence requires special care, and the presence of a division also complicates the matter. We explain hereafter how to cope with those issues.

For simplicity, we will make the following hypotheses throughout this section, which correspond to the standard ones in the Newton-Hensel method. They are that the starting $x_{0}$ and $B_{0}$ are in a basin of convergence at least linear. This allows us to replace any encountered $x_{n}$ by its lift $\tilde{x}_{n}$ to a higher precision (and same for $B_{n}$ ). Indeed, $\tilde{x}_{n}$ will still be in the basin of convergence and then follows the same convergence property. These liftings allow to mitigate the fact that some divisions are reducing the amount of precision so that only arbitrary added digits are destroyed by the divisions. ${ }^{6}$

Assumption 6.1. We assume that $x_{0}$ and $x^{\star}$ are in $O_{K}$, and that $\left\|f^{\prime}\left(x^{\star}\right)\right\|=\left\|f^{\prime}\left(x^{\star}\right)^{-1}\right\|=\left\|B_{0}\right\|=\left\|B_{0}^{-1}\right\|=1$. We also assume that some $\rho_{1} \leq 1$ and $\rho_{2} \leq 1$ are given such that $B\left(x^{\star}, \rho_{1}\right) \times$ $B\left(f^{\prime}\left(x^{\star}\right), \rho_{2}\right)$, is a basin of convergence at least linear and for any $x \in B\left(x^{\star}, \rho_{1}\right)$, and $\rho \leq \rho_{1}, f(x+B(0, \rho))=f(x)+f^{\prime}\left(x^{\star}\right) \cdot B(0, \rho)$ (see the Precision Lemma 3.16 of [9])

The assumption on $B_{0}$ and $f^{\prime}\left(x^{\star}\right)$ states that they are unimodular, which is the best one can assume regarding to conditioning and precision. Indeed if $M \in G L_{m}(K)$ is unimodular $\left(\|M\|=\left\|M^{-1}\right\|=\right.$ 1), then for any $x \in K^{m},\|M x\|=\|x\|$. Over $\mathbb{Q}_{p}, M \in M_{m}\left(\mathbb{Z}_{p}\right)$ is unimodular if and only if its reduction in $M_{m}(\mathbb{Z} / p \mathbb{Z})$ is invertible (and idem for $\mathbb{Q} \llbracket T \rrbracket$ and $\mathbb{Q}$ ). The last assumption is there to provide the precision on the evaluations $f\left(x_{k}\right)$ 's. It is satisfied if $f \in O_{K}\left[X_{1}, \ldots, X_{m}\right]$.

Precision and complexity settings. Let $M(N)$ be a superadditive upper-bound on the arithmetic complexity over the residue field of $O_{K}$ for the computation of the product of two elements in $O_{K}$ at precision $O\left(\pi^{N}\right)$, and $L$ be the size of a straight-line program that computes the system $f$. One can take $M(N) \in \tilde{O(N)}$.

Working over $K$ with zealous arithmetic, the ultrametric counterpart of interval arithmetic $[9, \S 2.1]$, the interval of integers $\llbracket a, b \llbracket$ indicates the coefficients of an element $x \in K$ represented in the computer as $x=\sum_{i=a}^{b-1} x_{i} \pi^{i}$, with $x_{i} \in O_{K} /\langle\pi\rangle$. In this way $\operatorname{val}(x)=a$, its absolute precision is $\operatorname{abs}(x)=b$, and its relative precision is $\operatorname{rel}(x)=b-a$. We recall the usual precision formulae, and assume in the algorithm below that it is how the software manages zealous arithmetic (as in Magma, SageMath, Pari). See loc. cit. for more details.

$$
\begin{aligned}
\llbracket a, b \llbracket \times \llbracket c, d \llbracket & =\llbracket a+c, \min (a+d, b+c) \llbracket \\
\llbracket a, b \llbracket / \llbracket c, d \llbracket & =\llbracket a-c, \min (a+d-2 c, b-c) \llbracket
\end{aligned}
$$

The cost of multiplying two elements of relative precision $a$ and $b$ is within $M(\max (a, b))$, and to divide one by the other is in $4 \mathrm{M}(\max (a, b))+\max (a, b)[25, \operatorname{Thm} 9.4]$.

To perform changes in the precision, we use the same notation as Magma's function for doing so. If $x$ has interval $\llbracket a, b \llbracket$, the (destructive) procedure "ChangePrec $(\sim x, c)$ " either truncates $x$ to absolute precision $c$ if $c \leq b$, or lifts with zero coefficients $0 \pi^{b}+$ $\cdots+0 \pi^{c-1}$ to fit the interval $\llbracket a, c \llbracket$, if $c>b$. The non-destructive counterpart is denoted "ChangePrec $(x, c)$ " without .

\footnotetext{
${ }^{6}$ This an example of an adaptive method, which can also be used in Newton's method when divisions occur.
}

\subsection{Effective Broyden's method}

We start from an initial approximation $x_{0}$ at precision one, for example given by a modular method. The inverse of the Jacobian at precision one provides $B_{0}^{-1}$. It yields a cost of $O\left(m^{\omega}\right)$, but the complexity analysis of Remark 6.4 shows that it is negligible. Obtaining these data is not always obvious [12], but is the standard hypothesis in the context of modular methods. We write $v_{k}=\operatorname{val}\left(f_{k}\right)$,

In an ideal situation. Assume an oracle provides the valuations $v_{0}, v_{1}, v_{2}, \ldots, v_{n}, \ldots$ (computed by a Broyden method at arbitrarily large precision). From this ideal situation, we derive the simple and costless modifications required in reality. This analysis allows us to know how efficient can a Broyden method be, which is noteworthy for comparing it to Newton's. The implementation of Iteration $n$ ( $n=0$ included) follows the lines hereunder. The rightmost interval indicates the output interval precision of the object computed (following $(\mathrm{P})$ ), while the middle indicates a complexity estimate.

Input: (1) $B_{n}^{-1}$ has interval $\llbracket 0, v_{n} \llbracket$ and is unimodular.

$\overline{\text { (2) } x_{n}}$ has interval $\llbracket 0, v_{n}+v_{n+1} \llbracket$ (non-zero entries in $\llbracket 0, v_{n-1}+v_{n} \llbracket$ ).

(3) $f_{n}$ has interval $\llbracket v_{n}, v_{n}+v_{n+1} \llbracket$.

Output: (i) $B_{n+1}^{-1}$ with interval $\llbracket 0, v_{n+1} \llbracket,\left(\operatorname{val}\left(\operatorname{det}\left(B_{n}^{-1}\right)\right)=0\right)$.

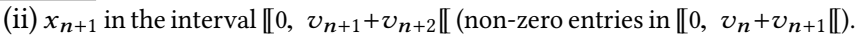

(iii) $f_{n+1}$ in the interval $\llbracket 0, v_{n+1}+v_{n+2} \llbracket$.

(1) ChangePrec $\left(\sim B_{n}^{-1}, v_{n+1}\right)$;

(2) $s_{n} \leftarrow-B_{n}^{-1} \cdot f_{n} ; \quad m^{2} \mathrm{M}\left(v_{n+1}\right)$

$\llbracket 0, v_{n+1} \llbracket$

(3) $x_{n+1} \leftarrow x_{n}+s_{n}$;

$\llbracket v_{n}, v_{n}+v_{n+1} \llbracket$

(4) ChangePrec $\left(\sim x_{n+1}, v_{n+1}+v_{n+2}\right)$;

$\llbracket 0, v_{n}+v_{n+1} \llbracket$

(5) $f_{n+1} \leftarrow f\left(x_{n+1}\right)$;

$$
L \cdot M\left(v_{n+1}+v_{n+2}\right) \quad \llbracket v_{n+1}, v_{n+1}+v_{n+2} \llbracket
$$

(6) $\overline{f_{n+1}} \leftarrow$ ChangePrec $\left(f_{n+1}, v_{n}+v_{n+1}\right)$; $\llbracket v_{n+1}, v_{n+1}+v_{n} \llbracket$

(7) $h_{n} \leftarrow B_{n}^{-1} \cdot \overline{f_{n+1}} ; \quad m^{2} \mathrm{M}\left(v_{n+1}\right) \quad \llbracket v_{n+1}, v_{n}+v_{n+1} \llbracket$

(8) $u_{n} \leftarrow$ Eq.(13); $\quad$ (negligible) $\llbracket-v_{n}, v_{n+1}-v_{n} \llbracket$

(9) $r_{n} \leftarrow u_{n}^{T}$. ChangePrec $\left(B_{n}^{-1}, v_{n}\right) ; m^{2} \mathrm{M}\left(v_{n+1}\right) \llbracket-v_{n}, 0 \llbracket$

(10) ChangePrec $\left(\sim \overline{f_{n+1}}, 2 v_{n}\right) ; \quad \llbracket v_{n+1}, 2 v_{n} \llbracket$

(11) den $\leftarrow 1+r_{n} \cdot \overline{f_{n+1}} ; \quad m \mathrm{M}\left(v_{n+1}\right) \quad \llbracket 0, v_{n} \llbracket$

(12) Num $\leftarrow h_{n} \cdot r_{n} ; \quad m^{2} \mathrm{M}\left(v_{n}\right) \quad \llbracket v_{n+1}-v_{n}, v_{n+1} \llbracket$

(13) $\mathrm{N}_{n} \leftarrow \mathrm{Num} / \mathrm{den} ; \quad 4 m^{2} \mathrm{M}\left(v_{n}\right) \quad \llbracket v_{n+1}-v_{n}, v_{n+1} \llbracket$

(14) $B_{n+1}^{-1} \leftarrow B_{n}^{-1}-\mathrm{N}_{n}$;

(15) return $B_{n+1}^{-1}, x_{n+1}, f_{n+1}$

$\llbracket 0, v_{n+1} \llbracket$

We emphasize again that thanks to the careful changes of precision undertaken, the precisions are automatically managed by the software, would it have zealous arithmetic implemented. It is then immediate to check that the output verifies the specifications. Moreover from the positive valuation of $\mathrm{N}_{n}$ it is clear that $B_{n+1}$ is unimodular. Thus Iteration $n+1$ can be initiated with these outputs.

Complexity of the ideal situation. The arithmetic cost of Iteration $n$ is within $\left(3 m^{2}+m\right) \mathrm{M}\left(v_{n+1}\right)+5 m^{2} \mathrm{M}\left(v_{n}\right)+L \cdot \mathrm{M}\left(v_{n+2}+v_{n+1}\right)$. If we assume an exponent of convergence $\alpha>1$, i.e. $v_{n+1} \approx \alpha v_{n}$ for "not too small" $n$, then the total cost to reach a precision $N \approx$ $\alpha^{\ell+1} \approx v_{\ell+1}$ ( $\ell$ steps, including a 0 -th one) is upper-bounded by

$$
\left(5 m^{2}+\left(3 m^{2}+m\right) \alpha^{2}+L(1+\alpha)^{2} \alpha^{2}\right) M(N /(\alpha-1))
$$

In reality. Using the same notations and inputs at Iteration $n$ as in the ideal situation above, what changes in reality is that while $v_{n}$ is known $v_{n+1}$ and $v_{n+2}$ are not, but are approximated by $\alpha v_{n} \geq$ 
$v_{n+1}$ and $\alpha^{2} v_{n} \geq v_{n+2}$ respectively, where $\alpha$ is fixed by the user Precisely, $B_{n}^{-1}$ and $x_{n}$ are known at the correct precision, but $f_{n}$ has an approximated interval $\llbracket 0, v_{n}+\alpha v_{n} \llbracket$. To minimize the overhead cost it induces compared to the ideal situation, once we know $v_{n+1}$ (Line 5) we insert some intermediate corrective steps denoted (5.1)-(5.5) thereafter, between Line (5) and Line (6); they require no arithmetic operations.

(5.1) ChangePrec $\left(\sim B_{n}^{-1}, v_{n+1}\right)$

(5.2) ChangePrec $\left(\sim s_{n}, v_{n}+v_{n+1}\right)$

(5.3) Tune $\alpha$ if necessary using the new ratio $\frac{v_{n+1}}{v_{n}}$

(5.4) ChangePrec $\left(\sim x_{n}, v_{n+1}+\alpha v_{n+1}\right)$

(5.5) ChangePrec $\left(\sim f_{n+1}, v_{n+1}+\alpha v_{n+1}\right)$

Most importantly, the remaining Lines (6)-(15) are not impacted since these computations involve now the known $v_{n+1}$ (and not the unknown $v_{n+2}$ ): the intervals, and thus costs obtained are the same as in the ideal situation. On the other hand, Lines (1)-(5) are performed as such with an overhead cost. Among them, only Lines (2), (5) have a non negligible cost. At Line (2), $B_{n}^{-1}$ has approximated interval «0, $\alpha v_{n} \llbracket$, yielding a cost of $m^{2} \mathrm{M}\left(\alpha v_{n}\right)$. At Line (5) $x_{n+1}$ has approximated interval [0, $v_{n}\left(\alpha+\alpha^{2}\right)$ [, yielding a cost of $\operatorname{LM}\left(v_{n}(\alpha(1+\alpha))\right)$. Thus the overhead cost "ovh $n$ " at Iteration $n$ is:

$$
m^{2}\left(\mathrm{M}\left(\alpha v_{n}\right)-\mathrm{M}\left(v_{n+1}\right)\right)+L\left(\mathrm{M}\left(v_{n} \alpha(1+\alpha)\right)-\mathrm{M}\left(v_{n+1}+v_{n+2}\right)\right)
$$

This quantity depends on the gaps $\alpha v_{n}-v_{n+1}$ and $\alpha^{2} v_{n}-v_{n+2}$. These gaps increase with $n$, but, thanks to the tuning of Step (5.3), reasonably at a linear rate:

Assumption 6.2. The "error gap" $\left|\alpha v_{n}-v_{n+1}\right|=O(n)$.

Under this assumption it is easy to (crudely) bound $\sum_{n=0}^{\ell+1} \mathrm{ovh}_{n}$ of Eq. (34) by $\left(L+m^{2}\right) O(N \log (N))$. Being independent on $\alpha$ this is negligible in front of $O\left(L+m^{2}\right) \mathrm{M}\left(\frac{N}{\alpha-1}\right)$ for $\alpha<2$. The theorem below wraps up the considerations made above with Eq. (33):

THEOREM 6.3. If Broyden's method has $Q$-order of convergence $\alpha$ on $B\left(x^{\star}, \rho_{1}\right) \times B\left(f^{\prime}\left(x^{\star}\right), \rho_{2}\right)$, then under Assumption 6.1 and 6.2, the cost of computing $x^{\star}+O\left(\pi^{N}\right)$ is in $O\left(\left(m^{2}+L\right)\right) \mathrm{M}\left(\frac{N}{\alpha-1}\right)$.

Remark 6.4. Understanding the $Q$-order of convergence is a major and notoriously difficult problem in the numerical analysis community. Numerical evidence shows it deteriorates with $m$, and is larger than $2^{1 / 2 m}$ (Theorems 4.7-4.8). Some experiments suggest that taking $\alpha \approx 2^{1 / m}$ is not unreasonable. We then get a cost in $O\left(\left(m^{2}+L\right) M\left(\frac{N}{\alpha-1}\right)\right) \approx O\left(\left(m^{2}+L\right) M(N m)\right)$. For comparison, denoting $\omega<3$ the exponent of the cost of matrix product, the standard analysis of Newton's method for rational fractions would lead to $O\left(\left(m^{\omega}+m L\right) M(N)\right)$. Consequently, in this setting, for large $m$, there is little hope that Broyden's method can outperform Newton's when both are available. Remember though other worthwile applications in the paragraph "Motivations" in Introduction.

\section{NUMERICAL DATA}

An implementation of our ultrametric Broyden method in Magma [4] with more data is available at http://xdahan.sakura.ne.jp/broyden20. html. We report the data obtained using the three families of systems, derived from page 36 of [18]. The families are indexed by $t \in \pi O_{K}$ :
- $F_{1}=\left(\left(x_{1}-1\right)^{2}+\left(x_{2}-1\right)^{2}-4-t x_{1} x_{2}-t^{2} x_{1},\left(x_{1}+1\right)^{2}+\left(x_{2}+1\right)^{2}-4-t x_{1}\right)$ in $K\left[x_{1}, x_{2}\right]$.

- $F_{2}=\left(\left(x_{1}-1\right)^{2}+\left(x_{2}-1\right)^{2}+\left(x_{3}-1\right)^{2}-5-t-t^{2},\left(x_{1}+1\right)^{2}+\left(x_{2}+1\right)^{2}+\right.$ $\left.\left(x_{3}+1\right)^{2}-5-t, 2 x_{1}^{2}+x_{2}^{2}+x_{3}^{2}-3-t^{2}\right)$ in $K\left[x_{1}, x_{2}, x_{3}\right]$.

- $F_{3}=\left(\left(x_{1}-1\right)^{2}+\left(x_{2}-1\right)^{2}+\left(x_{3}-1\right)^{2}+\left(x_{4}-1\right)^{2}-8-t-t^{2},\left(x_{1}+\right.\right.$ $1)^{2}+\left(x_{2}+1\right)^{2}+\left(x_{3}+1\right)^{2}+\left(x_{4}+1\right)^{2}-8-t, 2 x_{1}^{2}+x_{2}^{2}+x_{3}^{2}+x_{4}^{2}-5-$ $\left.t^{2}, 2 x_{1} x_{2}+x_{3} x_{2}-2 x_{3} x_{4}+2 x_{4} x_{1}+3-t^{2}\right)$ in $K\left[x_{1}, x_{2}, x_{3}, x_{4}\right]$.

Valuation of $f\left(x_{k}\right)$ and numerical estimation of the order of Qconvergence for $\mathbb{Q} \llbracket T \rrbracket$ are compiled in the following graphic. For $K=\mathbb{Q}_{p}$, and $\mathbb{F}_{p} \llbracket t \rrbracket$ with $p=17$ we experienced the same behaviour.

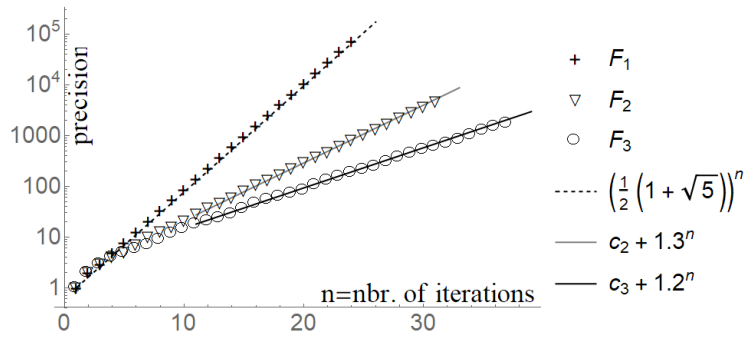

\section{REFERENCES}

[1] Eric Bach. Iterative root approximation in p-adic numerical analysis. fournal of Complexity, 25(6):511-529, 2009.

[2] Walter Baur and Volker Strassen. The complexity of partial derivatives. Theoretical computer science, 22(3):317-330, 1983.

[3] Jérémy Berthomieu, Joris Van Der Hoeven, and Grégoire Lecerf. Relaxed algorithms for p-adic numbers. F. Théor. Nombres Bordeaux, 23(3):541-577, 2011.

[4] Wieb Bosma, John Cannon, and Catherine Playoust. The Magma algebra system. I. The user language. 7. Symbolic Computation, 24(3-4):235-265, 1997.

[5] Richard P Brent and Hsiang T Kung. Fast algorithms for manipulating formal power series. Fournal of the ACM (JACM), 25(4):581-595, 1978.

[6] Charles G Broyden. A class of methods for solving nonlinear simultaneous equations. Mathematics of computation, 19(92):577-593, 1965

[7] Charles G. Broyden, John E Dennis Jr, and Jorge J Moré. On the local and superlinear convergence of quasi-newton methods. IMA Journal of Applied Mathematics, 12(3):223-245, 1973.

[8] Richard L. Burden and J. Douglas Faires. Numerical analysis. Brooks/Cole, Cengage Learning, 9 edition, 2011.

[9] Xavier Caruso. Computations with $p$-adic numbers. Les cours $d u$ CIRM, 5(1):1-75, 2017.

[10] John E Dennis, Jr and Jorge J Moré. Quasi-newton methods, motivation and theory. SIAM review, 19(1):46-89, 1977.

[11] Michael J. Fischer and Larry J. Stockmeyer. Fast on-line integer multiplication. fournal of Computer and System Sciences, 9(3):317 - 331, 1974.

[12] Aviezri S. Fraenkel and Yaacov Yesha. Complexity of solving algebraic equations. Information Processing Letters, 10(4):178 - 179, 1980.

[13] David M Gay. Some convergence properties of broyden's method. SIAM fournal on Numerical Analysis, 16(4):623-630, 1979.

[14] C. Tim Kelley and Ekkehard W. Sachs. Broyden's method for approximate solution of nonlinear integral equations. The fournal of Integral Equations, 9(1):25-43, 1985.

[15] C. Tim Kelley and Ekkehard W. Sachs. Approximate quasi-Newton methods. Mathematical Programming, 48(1-3):41-70, 1990.

[16] Hsiang T Kung. On computing reciprocals of power series. Numerische Mathematik, 22(5):341-348, 1974

[17] Hsiang T Kung and Joseph Frederick Traub. All algebraic functions can be computed fast. Fournal of the ACM (FACM), 25(2):245-260, 1978.

[18] Grégoire Lecerf. Une alternative aux méthodes de réécriture pour la résolution des systèmes algébriques. PhD thesis, École polytechnique, France, 2001.

[19] José Mario Martínez. Practical quasi-newton methods for solving nonlinear systems. Journal of Computational and Applied Mathematics, 124(1):97 - 121, 2000. Numerical Analysis 2000. Vol. IV: Optimization and Nonlinear Equations.

[20] James M Ortega and Werner C Rheinboldt. Iterative solution of nonlinear equations in several variables, volume 30. Siam, 1970.

[21] Jean-Pierre Serre. A course in arithmetic. Springer GTM 7, 1973.

[22] Jack Sherman and Winifred J Morrison. Adjustment of an inverse matrix corresponding to a change in one element of a given matrix. The Annals of Mathematical Statistics, 21(1):124-127, 1950.

[23] Joris van der Hoeven. Relax, but don't be too lazy. Fournal of Symbolic Computation, 34(6):479 - 542, 2002. 
[24] Joris van der Hoeven. Newton's method and FFT trading. fournal of Symbolic Computation, 45(8):857-878, 2010.

[25] Joachim von zur Gathen and Jürgen Gerhard. Modern computer algebra. Cambridge University Press, New York, NY, USA, 2003. Second Edition. 